\title{
Transcriptome Analysis of Environmental Pseudomonas Isolates Reveals Mechanisms of Biodegradation of Naphthenic Acid Fraction Compounds (NAFCs) in Oil Sands Tailings
}

\author{
Parisa Chegounian ${ }^{1}$, Stephane Flibotte ${ }^{2}$, Kerry Peru ${ }^{3}{ }^{\circledR}$, John Headley ${ }^{3}$, Dena McMartin ${ }^{4}$, Bryne Gramlich ${ }^{5}$ \\ and Vikramaditya G. Yadav $1,6, *$ (D) \\ 1 Department of Chemical and Biological Engineering, The University of British Columbia, \\ Vancouver, BC V6T 1Z3, Canada; parisach@mail.ubc.ca \\ 2 UBC/LSI Bioinformatics Facility, University of British Columbia, Vancouver, BC V6T 1Z3, Canada; \\ stephane.flibotte@ubc.ca \\ 3 Watershed Hydrology and Ecology Research Division, Water and Science Technology Directorate, \\ Environment \& Climate Change Canada, Saskatoon, SK S7N 3H5, Canada; kerry.peru@canada.ca (K.P.); \\ John.Headley@canada.ca (J.H.) \\ 4 Department of Civil, Geological and Environmental Engineering, University of Saskatchewan, \\ Saskatoon, SK S7N 5A9, Canada; dena.mcmartin@usask.ca \\ 5 Allonnia L.L.C., Boston, MA 02210, USA; bgramlich@allonnia.com \\ check for \\ updates \\ 6 School of Biomedical Engineering, The University of British Columbia, Vancouver, BC V6T 1Z3, Canada \\ * Correspondence: vikramaditya.yadav@ubc.ca
}

Citation: Chegounian, P.; Flibotte, S.; Peru, K.; Headley, J.; McMartin, D.; Gramlich, B.; Yadav, V.G. Transcriptome Analysis of Environmental Pseudomonas Isolates Reveals Mechanisms of Biodegradation of Naphthenic Acid Fraction Compounds (NAFCs) in Oil Sands Tailings. Microorganisms 2021, 9, 2124. https://doi.org/10.3390/ microorganisms 9102124

Academic Editors: Julia M. Foght and Tariq Siddique

Received: 2 September 2021

Accepted: 25 September 2021

Published: 9 October 2021

Publisher's Note: MDPI stays neutral with regard to jurisdictional claims in published maps and institutional affiliations.

Copyright: () 2021 by the authors Licensee MDPI, Basel, Switzerland. This article is an open access article distributed under the terms and conditions of the Creative Commons Attribution (CC BY) license (https:// creativecommons.org/licenses/by/ $4.0 /)$.

\begin{abstract}
Naphthenic acid fraction compounds (NAFCs) are highly recalcitrant constituents of oil sands tailings. Although some microorganisms in the tailings can individually and synergistically metabolize NAFCs, the biochemical mechanisms that underpin these processes are hitherto unknown. To this end, we isolated two microorganisms, Pseudomonas protegens and Pseudomonas putida, from oils sands tailings and analyzed their transcriptomes to shed light on the metabolic processes employed by them to degrade and detoxify NAFCs. We identified 1048, 521 and 1434 genes that are upregulated in P. protegens, P. putida and a 1:1 co-culture of the strains, respectively. We subsequently enumerated the biochemical activities of enriched genes and gene products to reveal the identities of the enzymes that are associated with NAFC degradation. Separately, we analyzed the NAFCs that are degraded by the two pseudomonads and their 1:1 co-culture and determined the composition of the molecules using mass spectrometry. We then compared these molecular formulas to those of the cognate substrates of the enriched enzymes to chart the metabolic network and understand the mechanisms of degradation that are employed by the microbial cultures. Not only does the consortium behave differently than the pure cultures, but our analysis also revealed the mechanisms responsible for accelerated rate of degradation of NAFCs by the co-culture. Our findings provide new directions for engineering or evolving microorganisms and their consortia for degrading NAFCs more stably and aggressively.
\end{abstract}

Keywords: naphthenic acids; pseudomonads; biodegradation; transcriptomics; bioinformatics; microbial consortia

\section{Introduction}

The oil sands in the Athabasca region of northern Alberta is an unconventional and plentiful energy reserve [1]. The mining and extraction of bitumen from these deposits consumes copious quantities of water. Conversely, bitumen extraction also generates a large volume of wastewater called oil sands process-affected water (OSPW) [2]. This waste stream is chronically and acutely toxic to aquatic life [3-7], and the principal toxicants in OSPW are a large group of organic molecules called naphthenic acids fraction compounds (NAFCs). The general formula of NAFCs is $\mathrm{C}_{\mathrm{c}} \mathrm{H}_{\mathrm{h}} \mathrm{N}_{\mathrm{n}} \mathrm{O}_{\mathrm{o}} \mathrm{S}_{\mathrm{s}}$ and their carbon content ranges 
between seven and 26 atoms [8-10]. OSPW contains several hundred NAFCs that can be further divided into $\mathrm{O}_{\mathrm{o}}, \mathrm{N}_{\mathrm{n}} \mathrm{O}_{\mathrm{o}}, \mathrm{O}_{\mathrm{o}} \mathrm{S}_{\mathrm{s}}, \mathrm{N}_{\mathrm{n}} \mathrm{S}_{\mathrm{s}}$ and $\mathrm{N}_{\mathrm{n}} \mathrm{O}_{\mathrm{o}} \mathrm{S}_{\mathrm{s}}$ compounds [5,11]. Several of these molecules are unsaturated, and the degree of unsaturation of an NAFC can be inferred from its double bound equivalents (DBE) [12,13], which is estimated as $c+\frac{1}{2}(n-h)+1$. Most of the debate surrounding the toxicity of OSPW in the popular press, however, has centered on a specific sub-group of NAFCs known as classic naphthenic acids (NAs). These molecules have an empirical formula of $\mathrm{C}_{\mathrm{C}} \mathrm{H}_{\mathrm{h}} \mathrm{O}_{2}$ and have been determined by environmental researchers to be the most toxic NAFCs [14-16]. Current environmental regulations forbid direct discharge into surrounding bodies of water $[17,18]$, and the OSPW is stored on-site in large tailings ponds while it awaits detoxification. However, NAFCs are notoriously recalcitrant, which makes OSPW very difficult to treat effectively and economically. As a consequence, the volume of untreated OSPW in the tailings ponds is continually rising and the inventory of water now exceeds 700 billion liters. This large volume of toxic water has amplified concerns about seepage into bodies of ground and surface water and contamination of sources of drinking water, not to mention the risks of a catastrophic spill. There is an unmet, urgent need for the development of wastewater treatment technologies that can economically rehabilitate OSPW [19].

The high concentration of NAFCs and other toxic compounds in the OSPW tailings ponds has sustained a unique ecological niche that abounds with microorganisms that have evolved to sense, uptake and metabolize these compounds and survive in this extreme environment [20-22]. Proteobacteria is the most abundant phylum in the microbiome in OSPW tailings ponds [14,23,24], and, herein, several microbial species of the genus Pseudomonas have been shown to degrade a subset of NAs and detoxify OSPW, albeit at exceedingly slow rates [16,25-29]. Microbial bioremediation clearly is a promising solution for decontamination and detoxification of OSPW. However, the range of structures and the rates at which NAFCs are degraded by these native microorganisms necessitates significant improvements in order for bioremediation to be sustainable and economical at scales required by oil sands operators [30-32]. Methodologies such as targeted genome engineering and adaptive laboratory evolution will be central to improving the substrate range and rate of biodegradation of NAFCs but these techniques require sound understanding of the biochemical pathways involved [32]. Unfortunately, the mechanisms of biodegradation of NAFCs are either poorly understood or have been delineated for idealized substrates [29,33-41]. In fact, the biodegradation pathways of most NAFCs in OSPW, notably $\mathrm{N}_{\mathrm{n}} \mathrm{O}_{\mathrm{o}}, \mathrm{O}_{\mathrm{o}} \mathrm{S}_{\mathrm{s}}, \mathrm{N}_{\mathrm{n}} \mathrm{S}_{\mathrm{s}}$, and $\mathrm{N}_{\mathrm{n}} \mathrm{O}_{\mathrm{o}} \mathrm{S}_{\mathrm{s}}$ compounds, are entirely unknown. Elucidation of the genes, enzymes and metabolic pathways responsible for adaptation to and degradation of NAFCs is necessary in order to effectively and economically remediate OSPW.

The first step in investigating the mechanisms of biodegradation of NAFCs is isolating and enriching appropriate microbial chassis that could serve as key constituents of the bioremediation platform. Next, analyzing the transcriptomic responses using RNA-seq of the microorganisms to exposure to NAFCs sheds light on the genes, enzymes and metabolic pathways responsible for adaptation to and degradation of the toxic compounds $[42,43]$. We previously isolated two pseudomonads, P. protegens and P. putida, that can metabolize NAFCs in OSPW by exploiting their ability to grow on these compounds as the sole source of carbon in plate and liquid cultures [44]. Chemical analyses of the culture medium before and after biological activity revealed that the two microorganisms exhibit unique propensities and rates for degrading NAFCs and that these differences are more pronounced for classic NAs. In the current study, we have employed RNA-seq to elucidate the mechanisms of biodegradation of NAFCs by individual and co-cultures of P. protegens and P. putida. Till date, no studies have been performed on transcriptomic response of microorganisms isolated from OSPW tailings ponds. In addition to deducing the transcriptional responses of $P$. protegens and P. putida, we also correlated this information to mass spectrometry data to identify NAFCs as substrates of individual enzymes. The substrates were then classified based on their heteroatom compositions and the enzyme-substrate pairs were then used to construct the biodegradation pathways. Finally, we also compared the metabolic networks 
of the two strains in individual and 1:1 co-culture with an eye on developing consortia for biodegradation.

\section{Materials and Methods}

\subsection{Extraction and Characterization of NAFCs}

OSPW samples were kindly provided by Suncor Energy. All chemicals and consumables used in this study were purchased from Fisher Scientific Canada (Ottawa, ON). We stored the samples in sealed polyethylene containers in the dark at $4{ }^{\circ} \mathrm{C}$. To test the samples, we first removed suspended particulate matter from $1 \mathrm{~L}$ batches of OSPW using vacuum filtration through grade- 4 glass fiber filters having a nominal particle retention size of $1.2 \mu \mathrm{m}$. The $\mathrm{pH}$ of the filtered water was then lowered to $\mathrm{pH} 2$ using $\mathrm{H}_{2} \mathrm{SO}_{4}$ and NAFCs were subsequently extracted with $100 \mathrm{~mL}$ of dichloromethane (DCM). The OSPW was treated twice with the same batch of DCM to maximize the concentration of the extracted NAFCs. For analytical characterization, the organic fraction was dissolved in a 1:1 solution (by volume) of acetonitrile and deionized water until the nominal concentration of NAFCs in the solution matched that of the original OSPW sample. We analyzed this mixture using HPLC-Orbitrap mass spectrometry. Details about the chromatography and mass spectrometry methods have been detailed in the accompanying Electronic Supporting Information (ESI) package.

\subsection{Genome Sequencing and Assembly}

We extracted high-molecular weight DNA from the water samples using chemical extraction. We verified the fragment size distribution, quantified the concentration of DNA and sequenced the libraries using the Oxford Nanopore and Illumina platforms. The methodology used for the genome assembly and the statistics (Table S1) have been summarized in the accompanying ESI package. We then used the Prokka package to predict and annotate the genes (Figure 1, yellow boxes) [45].

\subsection{Evaluating Biodegradation of NAFCs by the Microbial Cultures}

We once again filtered the OSPW using grade- 4 glass fiber filters having a nominal particle retention size of $1.2 \mu \mathrm{m}$ to eliminate particulate matter. Separately, we prepared starter cultures of $P$. putida and P. protegens by directly inoculating LB medium with glycerol stocks of the strains. The two cultures were propagated overnight at $22{ }^{\circ} \mathrm{C}$ under agitation at $200 \mathrm{rpm}$ and were then used to separately inoculate $50 \mathrm{~mL}$ of filtered OSPW in $250 \mathrm{~mL}$ shaker flasks. In addition to pure cultures of the two strains, we also prepared a 1:1 co-culture. All cultures had a starting optical density $\left(\mathrm{OD}_{600}\right)$ of 0.1 . The three cultures were then propagated for 30 days at $22{ }^{\circ} \mathrm{C}$ under constant agitation at $200 \mathrm{rpm}$. The extent of degradation of NAs by the cultures over the 30-day period was then quantified using HPLC-Orbitrap mass spectrometry. Additionally, we also resuspended the extracted NAFCs in deionized water to a level that matches their original concentration in OSPW. We subsequently assessed the toxicity (EC50) of the resuspended NAFC solution on Vibrio fischeri by quantifying the inhibition of growth over a $15 \mathrm{~min}$ duration [46].

\subsection{RNA Extraction, Library Preparation and Sequencing}

Three tester samples of M9 media supplemented with $280 \mathrm{mg} / \mathrm{L}$ of the extracted NAFCs were inoculated with P. putida, P. protegens and a 1:1 co-culture of the two strains. The starting $\mathrm{OD}_{600}$ in each culture was 0.1 . Driver samples were identically prepared, albeit without a carbon source. Suppression subtractive hybridization (SSH) was then employed to prepare libraries of transcripts associated with up-regulated genes in the tester samples compared to the corresponding driver samples [47]. Total RNA was isolated from all six testers and drivers using a PureLink ${ }^{\mathrm{TM}}$ RNA Mini Kit (Thermo Fisher Scientific, Ottawa, ON, Canada) by following the manufacturer's protocol. Subsequently, $2 \mu \mathrm{g}$ of RNA per sample was used to synthesize cDNA, amplify the up-regulated transcripts by $\mathrm{SSH}$, and generate RNA libraries using the PCR-Select ${ }^{\mathrm{TM}}$ cDNA Subtraction Kit (Takara 
Bio, San Jose, CA, USA). RNA-seq libraries were prepared thereafter and sequenced on the Illumina HiSeq platform using a paired protocol of $2 \times 150 \mathrm{bp}$ (Genewiz, Seattle, WA, USA). A cosmic summary of the sequencing results of the RNA-seq libraries has been provided in Table S2 in the ESI package.

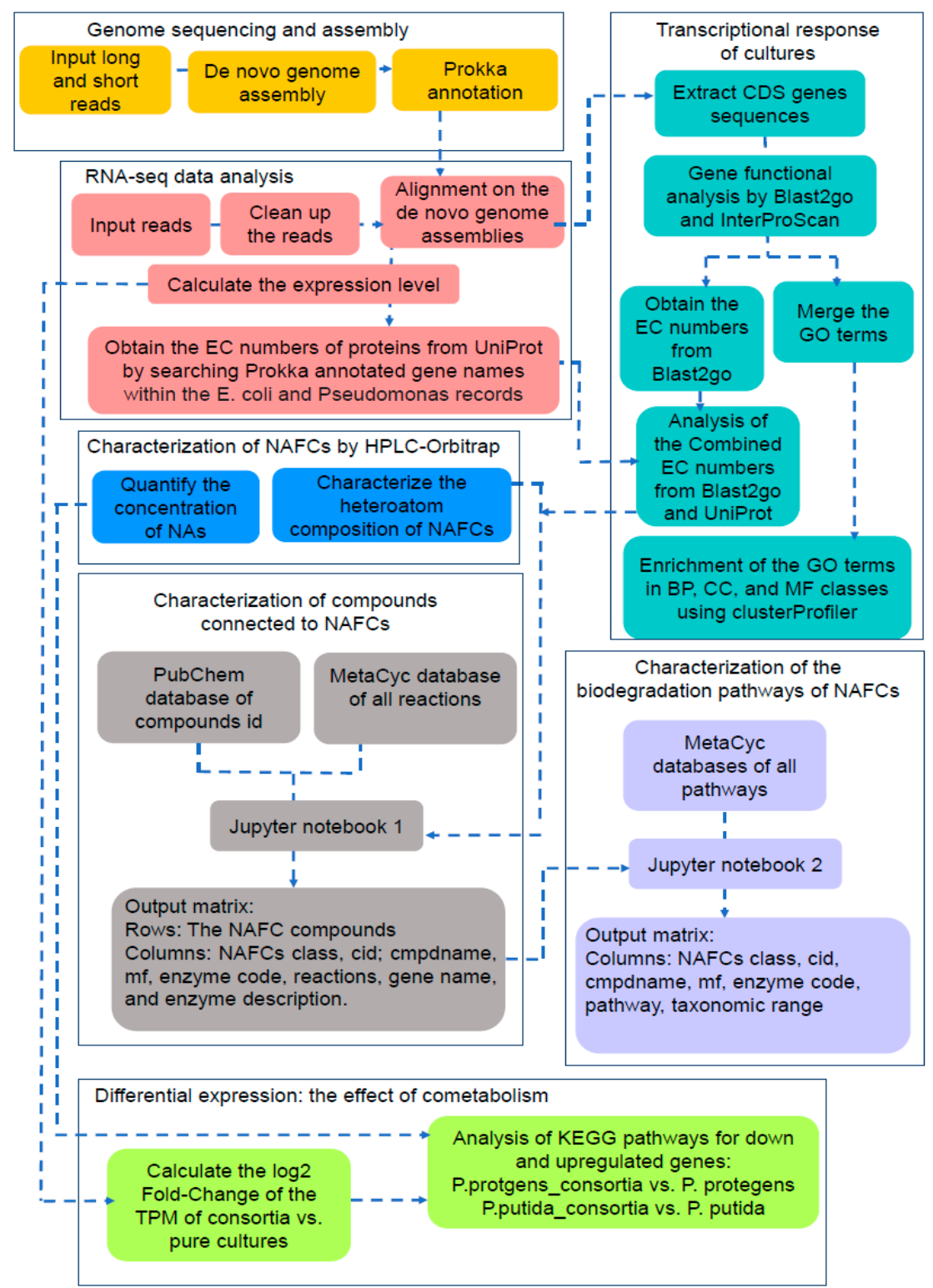

Figure 1. Data analysis workflow. Genome sequencing and assembly use long and short reads as inputs (yellow boxes). Short reads in the FastQ files of the RNA-seq libraries of pure P. putida, P. protegens and the 1:1 co-culture are aligned to the annotated genomes (red boxes). These alignments later serve as starting points for analyzing the transcriptional response of the microorganisms in either the pure cultures or co-culture by correlating chemical data (blue boxes) with GO terms and Enzyme Commission numbers (teal boxes). Cheminformatic analyses of the degraded NAFCs (grey boxes), which includes further categorization of the molecules by their class, PubChem ID (id), name (cmpdname) and molecular formula ( $\mathrm{mf}$ ), allows comparisons between the structures of the NAFCs and those of canonical substrates of enzymes determined previously (blue boxes). This step yields further information about the enzymatic reactions and their genes, which, in turn, reveals the biodegradation pathway (purple boxes). We also calculated fold-changes of the overexpressed and down-regulated genes (lime boxes) to provide insights about co-operative metabolism by the strains in the co-culture. 


\subsection{Analysis of the RNA-seq Data}

The reads were aligned to our de novo genome assemblies with the BWA tool [48]. The resulting bam files were sorted by coordinates and PCR duplicates were removed from the sorted reads using SAMtools. On the other hand, the RNA-seq library generated from the co-culture was aligned to a composite genome comprising of the genomes of P. putida and P. protegens. Read counts at the gene levels were then estimated for each RNA-seq library using the bedtools toolkit [49]. We then calculated the Transcripts Per Million (TPM) values using an in-house Perl script. We only retained genes that were covered by sequencing reads for at least 150 bases or $90 \%$ of their length (Figure 1, red boxes) for the subsequent analyses.

\subsection{Characterization of the Genes Involved in Biodegradation of NAFCs}

We characterized the transcriptional responses of the three cultures by determining the Gene Ontology (GO) annotations and Enzyme Commission (EC) numbers of the gene products that were previously identified using RNA-seq (Figure 1, teal boxes). Functional annotation of the genes for each RNA-seq library was performed using GO annotations with Blast2GO, InterProScan [50] and the nr database of the Pseudomonas genus with a BLAST expectation value of $1 \times 10^{-3}$. The GO terms from Blast2GO and InterProScan were then merged and used for characterization of the transcriptional responses of the Pseudomonas cultures to NAFCs. The statistical over-representation analysis for GO terms was performed using the universal enricher function of the clusterProfiler package from Bioconductor [51]. We focused on the biological processes (BPs) and molecular functions (MFs) clusters owing to the high likelihood of these genes being involved in biodegradation of NAFCs. Similarly, we assessed the Enzyme Commission (EC) numbers by cross-referencing the results of Blast2GO's EC mapping function with EC numbers of the products of E. coli and Pseudomonas spp. genes in the UniProt database that were annotated by Prokka. In order to determine the metabolic network responsible for biodegradation of NAFCs, we developed an entirely novel approach that co-analyses transcript and chemical data. The code for the analysis is included in the two Jupyter notebooks in the ESI package.

Briefly, the code in the first notebook matches the EC numbers of gene products identified from the RNA-seq libraries with enzymatic reactions in the MetaCyc database, fetches the Pubchem IDs of the substrates for the identified reactions and then retrieves their molecular formulas from the Pubchem database. Next, the algorithm classifies the retrieved molecular formulas into NAFC groups based on their heteroatom composition. These groups include $\mathrm{O}_{\mathrm{o}}, \mathrm{N}_{\mathrm{n}} \mathrm{O}_{\mathrm{o}}, \mathrm{O}_{\mathrm{o}} \mathrm{S}_{\mathrm{s}}, \mathrm{S}_{\mathrm{s}}, \mathrm{N}_{\mathrm{n}}, \mathrm{N}_{\mathrm{n}} \mathrm{O}_{\mathrm{o}} \mathrm{S}_{\mathrm{s}}$ and CH NAFCs. Differences between the substrates deduced for the pure cultures and co-cultures were assessed statistically with the aid of the Pearson's Chi square test for count data in R using the function chisq.test. The compounds identified using the aforementioned algorithm were subsequently inputted to the second Jupyter notebook. Due to over-generality of the integrated pathway maps on the Kyoto Encyclopedia of Genes and Genomes (KEGG) pathway database, we used more localized and individualized maps on MetaCyc to identify the NAFC degradation pathways. The algorithm finds and matches the chemical IDs (CID) of the substrates to EC numbers of putative enzymes, and later interrogates all combinations of CID-EC combinations in the entire MetaCyc database. This search outputs two data frames for the CID-EC pairs, one with and the other without pathway information. These data frames are re-classified into the dominant NAFC groups (e.g., O, N, NO, NOS, S and SO compounds, among others), which finally allows for the determination of the presence or absence of CIDEC pairs in specific pathways. We also investigated the effect of co-operative metabolism in the co-culture by calculating the fold-change of the TPM values for P. putida and P. putida when the strains are cultured individually or together. Specifically, we identified KEGG pathways in P. putida and P. protegens whose constituent enzymes exhibited fold-changes lower and greater than 1 in the pure cultures or co-culture. 


\section{Results}

\subsection{Biodegradation of NAFCs by the Pseudomonas Cultures}

We quantified the substrate range (Figure 2A) and extent (Figure 2B) of biodegradation of NAFCs in OSPW by pure cultures and a 1:1 co-culture of P. putida and P. protegens after a 30-day treatment. OSPW and all three microbially-treated water samples contain a significantly higher fraction of $\mathrm{O}_{2}, \mathrm{O}_{3}$ and $\mathrm{O}_{4}$ NAFCs compared to other oxygenated species. Additionally, with the exceptions of $\mathrm{SO}_{2}$ and $\mathrm{SO}_{3}$ species, the proportion of $\mathrm{S}$ - and $\mathrm{N}$-containing NAFCs is also relatively low in all four samples. This chemical distribution is consistent with previous reports. Additionally, $\mathrm{O}_{7}, \mathrm{O}_{8}, \mathrm{~N}_{2} \mathrm{O}_{2}$, and $\mathrm{S}_{2} \mathrm{O}_{3}$ groups do not occur in OSPW but are generated by microbial metabolism.

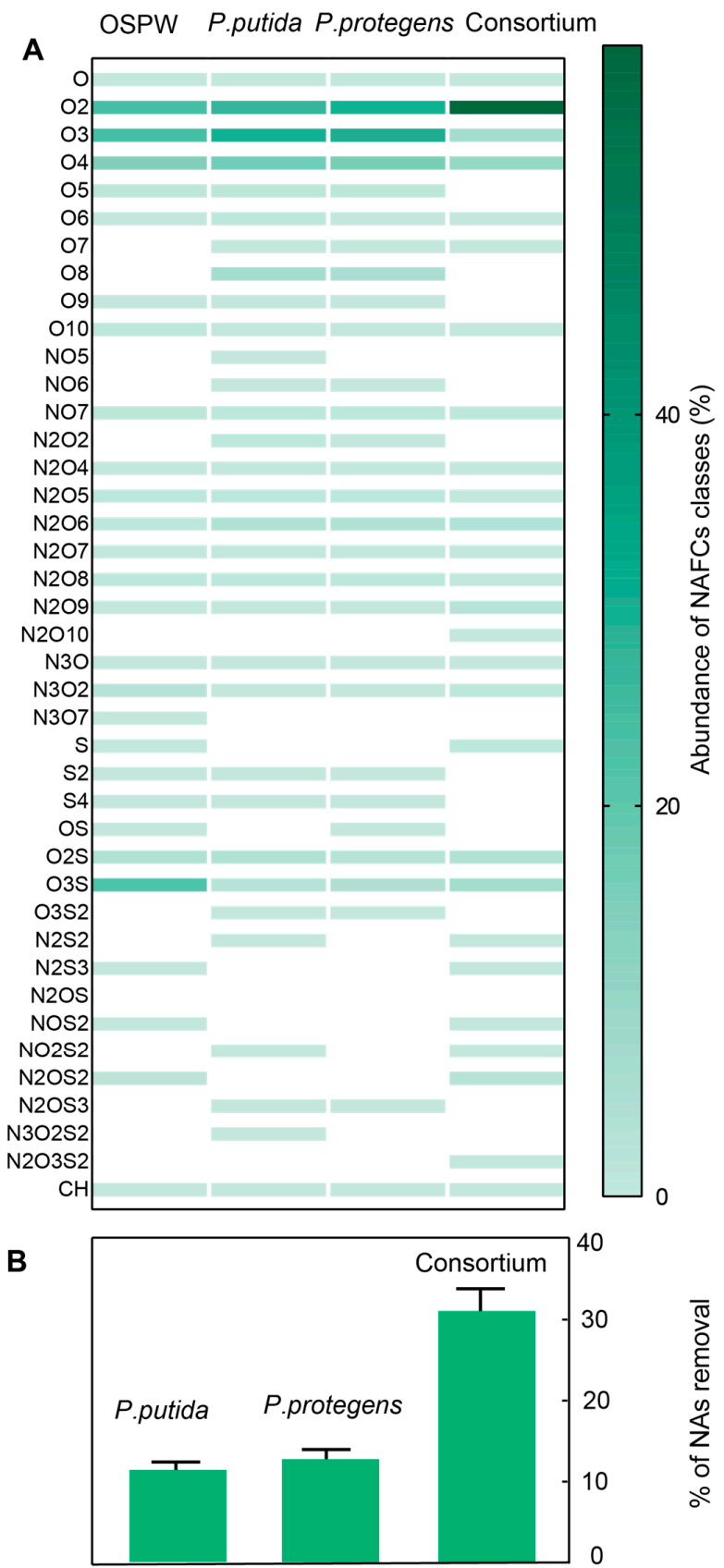

Figure 2. HPLC-Orbitrap results. (A) We quantified the relative abundance of the different classes NAFCs in OSPW before and after degradation by pure cultures and a 1:1 co-culture of P. putida and P. protegens. (B) We also estimated the total amount of NAs removed by the three cultures. NAs are the major toxicants in OSPW. 
On the other hand, $\mathrm{S}, \mathrm{N}_{2} \mathrm{~S}_{3}, \mathrm{NOS}_{2}$ and $\mathrm{N}_{2} \mathrm{OS}_{2}$ NAFCs are completely removed from OSPW after biodegradation. We speculate that these compounds are consumed by microorganisms as a source of sulfur. We also quantified the concentration of classic NAs in OSPW and their extent of removal by microbial activity. NAs are the major toxicants in OSPW [5]. We identified as many as 131 unique compounds that had a cumulative concentration of $28.2 \mathrm{mg} / \mathrm{L}$. Their carbon numbers and DBEs ranged between 5-25 and $1-10$, respectively. Of these, species containing 16 carbon atoms comprised $20.1 \%$ of the population of classic NAs, whereas compounds with a DBE of 4 accounted for $21.3 \%$ of the population. Aliphatic compounds most commonly have a DBE of 1 , whereas the DBEs of alicyclic compounds range between $2-4$. The raw gas chromatograms revealed that aliphatic and alicyclic NAs exhibit longer column retention times compared to aromatic and polycyclic compounds in our experiment. The pure cultures of P. putida, P. protegens and the $1: 1$ co-culture reduced the concentration of NAs in the culture media by $11 \%, 12 \%$ and $31 \%$, respectively (Figure $2 \mathrm{~B}$ ). These extents of degradation are roughly twice that of values detected by other groups, albeit using different, possibly niche Pseudomonas isolates [28]. We also plotted the difference between the concentrations of an individual NAFCs before $\left(C_{i o}\right)$ and after $\left(C_{i}\right)$ microbial treatment as a heat map for each of the three cultures (Figure S1 in the ESI package).

The plot confirms that the degradation profiles of the three cultures is distinct, which has implications for designing consortia. Additionally, we also observed that the concentrations of some NAFCs increased after microbial treatment, which reveals these species to be products of the degradation of other NAFCs. Finally, while the resuspended solution of extracted NAFCs elicited an average EC50 value of $82 \%$, treatment with pure cultures of P. putida, P. protegens and the 1:1 co-culture reduced the toxicity of the solution to EC50 values of $94 \%, 92 \%$ and $100 \%$, respectively.

\subsection{Transcriptional Responses of Pure Cultures of the Pseudomonas Isolates to NAFCs}

$\mathrm{SSH}$ is a simple and effective technique for generating cDNA that is highly enriched in differentially expressed genes that are present in either high or low abundance. The methodology permits simultaneous normalization and subtraction, which facilitates identification of over-expressed genes even for rare transcripts. The latter is critical for identification of novel genes that are selectively expressed upon exposure to NAFCs. The combination of high levels of enrichment, low background and normalized abundance of cDNA in the subtracted mixtures is ideal for rapid cloning of cDNA of the differentially expressed genes. Exposure to NAFCs elicited the expression of 1048 and 521 genes in pure cultures of P. protegens and P. putida, respectively. A total of 1434 genes were differentially expressed by cells in the 1:1 co-culture, of which 993 genes were expressed by P. protegens. It is notable that $P$. protegens induces many more genes than P. putida either separately or in co-cultures.

Although GO terms for cellular component (CC), biological process (BP), and molecular function $(\mathrm{MF})$ were statistically over-represented $(\mathrm{P}$-adj $<0.05)$ in all the cultures, we only considered the BP and MF classes for further analyses owing to the greater likelihood of their involvement in the biodegradation of NAFCs. We have summarized the enriched GO terms in Table S3 in the ESI. In total, 11 GO terms were significantly over-represented in the BP class (Figure 3), of which 4 GO terms, namely organonitrogen compound biosynthetic process (GO:1901566), amide biosynthetic process (GO:0043604), protein metabolic process (GO:0019538) and peptide metabolic process (GO:0006518) were common to all the cultures. Five GO terms corresponding to carbohydrate derivative metabolic process (GO:1901135), purine-containing compound biosynthetic process (GO:0072522), primary metabolic process (GO:0044238), macromolecule metabolic process (GO:0043170) and nitrogen compound metabolic process (GO:0006807) were uniquely enriched in the cultures of P. protegens. On the other hand, glutamine metabolic process (GO:0006541) was the only unique GO term that was enriched in cultures of $P$. putida. The GO terms for organonitrogen compound metabolic process (GO:1901564) was only biological process that was uniquely over-represented in both pure cultures. Similarly, GO terms in the MF class 
corresponding to heterocyclic compound binding (GO:1901363), organic cyclic compound binding (GO:0097159) and ligase activity (GO:0016874) were enriched in both pure cultures but no terms were uniquely enriched in the 1:1 co-culture.

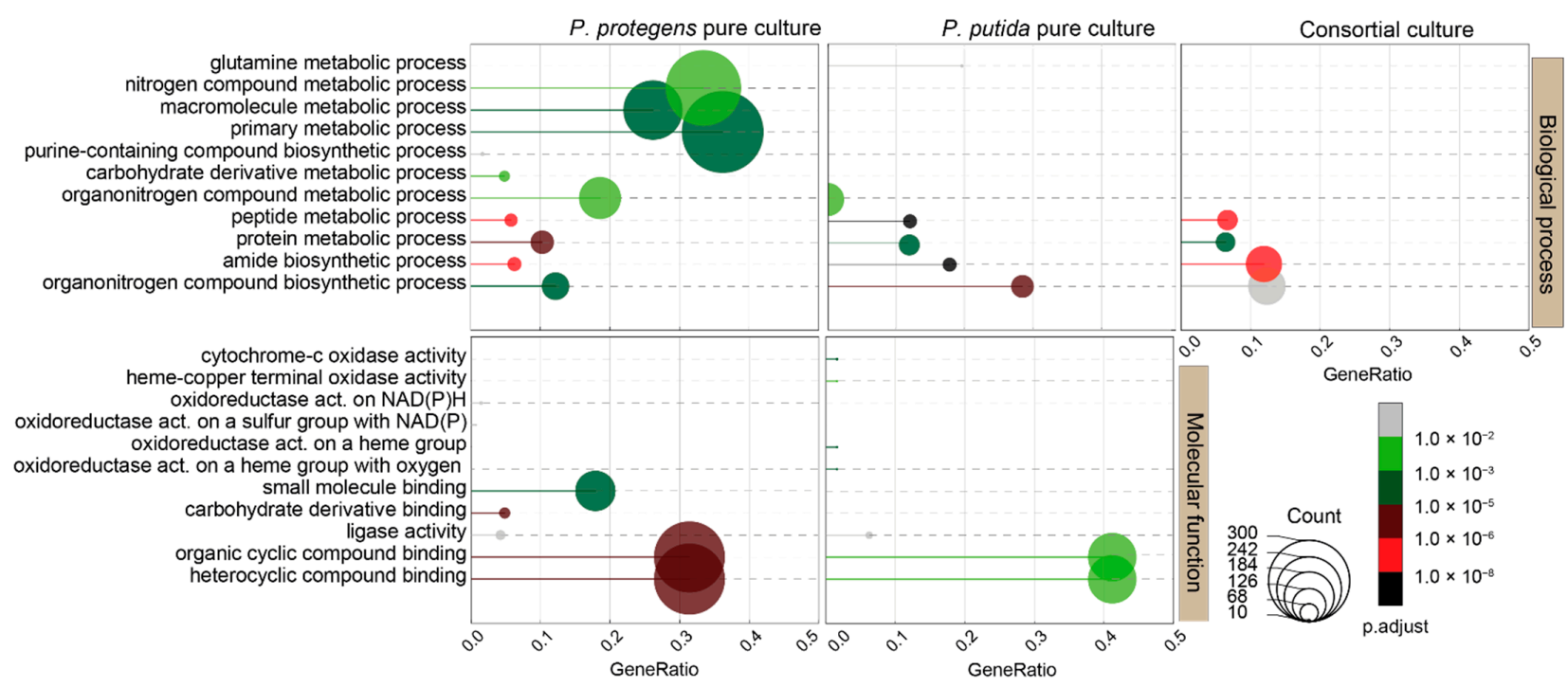

Figure 3. Over-representation of GO terms in pure and co-cultures of P. protegens and P. putida. We obtained enriched GO terms using Blast2GO, InterProScan and the nr database of the Pseudomonas genus with a BLAST expectation value of $1 \times 10^{-3}$, and then merged these results to calculate the GeneRatios (number of genes that over-expressed compared to the number of genes with the same term in the whole genome), adjusted p-values and the number of genes that were enriched in the cultures.

The pure cultures of P. protegens uniquely exhibited GO terms for carbohydrate derivative binding (GO:0097367), small molecule binding (GO:0036094), oxidoreductase activity on a sulfur group of donors with $\mathrm{NAD}(\mathrm{P})$ as acceptor (GO:0016668) and oxidoreductase activity acting on $\mathrm{NAD}(\mathrm{P}) \mathrm{H}(\mathrm{GO}: 0016651)$. GO terms corresponding to oxidoreductase activity on a heme group of donors with oxygen as acceptor (GO:0016676), oxidoreductase activity on a heme group of donors (GO:0016675), heme-copper terminal oxidase activity (GO:0015002), and cytochrome-c oxidase activity (GO:0004129) were uniquely enriched in pure cultures of P. putida.

We consolidated the results from Blast2GO and UniProt using EC numbers. This analysis yielded a total of 364, 198 and 463 over-expressed gene products in the cultures of $P$. protegens, P. putida, and the 1:1 co-culture (Table 1). The results suggest that P. putida leverages consortial metabolism to its advantage. The strain expresses fewer enzymes in the co-culture compared to pure cultures with the sole exception of hydrolases, which are greatly overexpressed in the co-culture. The biological significance of differential expression of hydrolases needs to be probed further. Additionally, in lieu of replication of the transcriptomics experiment, we confirmed phenotypic reproducibility by repeating the experiment to quantify biodegradation of NAFCs well over a dozen times. We observed a low standard deviation in the total amount of degradation (Figure 2) and nearly identical degradation patterns in the carbon numbers and DBEs of the NAFCs (Figure S1).

\subsection{Assessing the Similarity between Substrates of the Expressed Enzymes and NAFCs}

We identified the canonical substrates of the enzymes expressed by the two strains in each pure culture and the 1:1 co-culture by cross-referencing their EC numbers in the MetaCyc database. We subsequently filtered the results using a number of molecular descriptors. We observed that the distribution of substrates within the different classes of the NAFCs is significantly different between each pure culture and the 1:1 co-culture (Figure 4), with $p$-values of 0.01 and 0.03 for P. protegens and P. putida, respectively. 
Table 1. Summary of the number of genes (categorized based on enzyme class) that are expressed by the three cultures in response to exposure to NAFCs.

\begin{tabular}{ccccc}
\hline Enzyme Class & P. putida & $\begin{array}{c}\text { P. putida } \\
\text { in Co-Culture }\end{array}$ & P. protegens & $\begin{array}{c}\text { P. protegens } \\
\text { in Co-Culture }\end{array}$ \\
\hline EC 1-Oxidoreductases & 41 & 25 & 72 & 62 \\
EC 2-Transferases & 54 & 40 & 122 & 126 \\
EC 3 - Hydrolases & 70 & 77 & 276 & 305 \\
EC 4-Lyases & 15 & 6 & 23 & 25 \\
EC 5-Isomerases & 15 & 6 & 18 & 19 \\
EC 6-Ligases & 21 & 10 & 33 & 19 \\
EC 7-Translocases & 16 & 6 & 23 & 17 \\
\hline
\end{tabular}

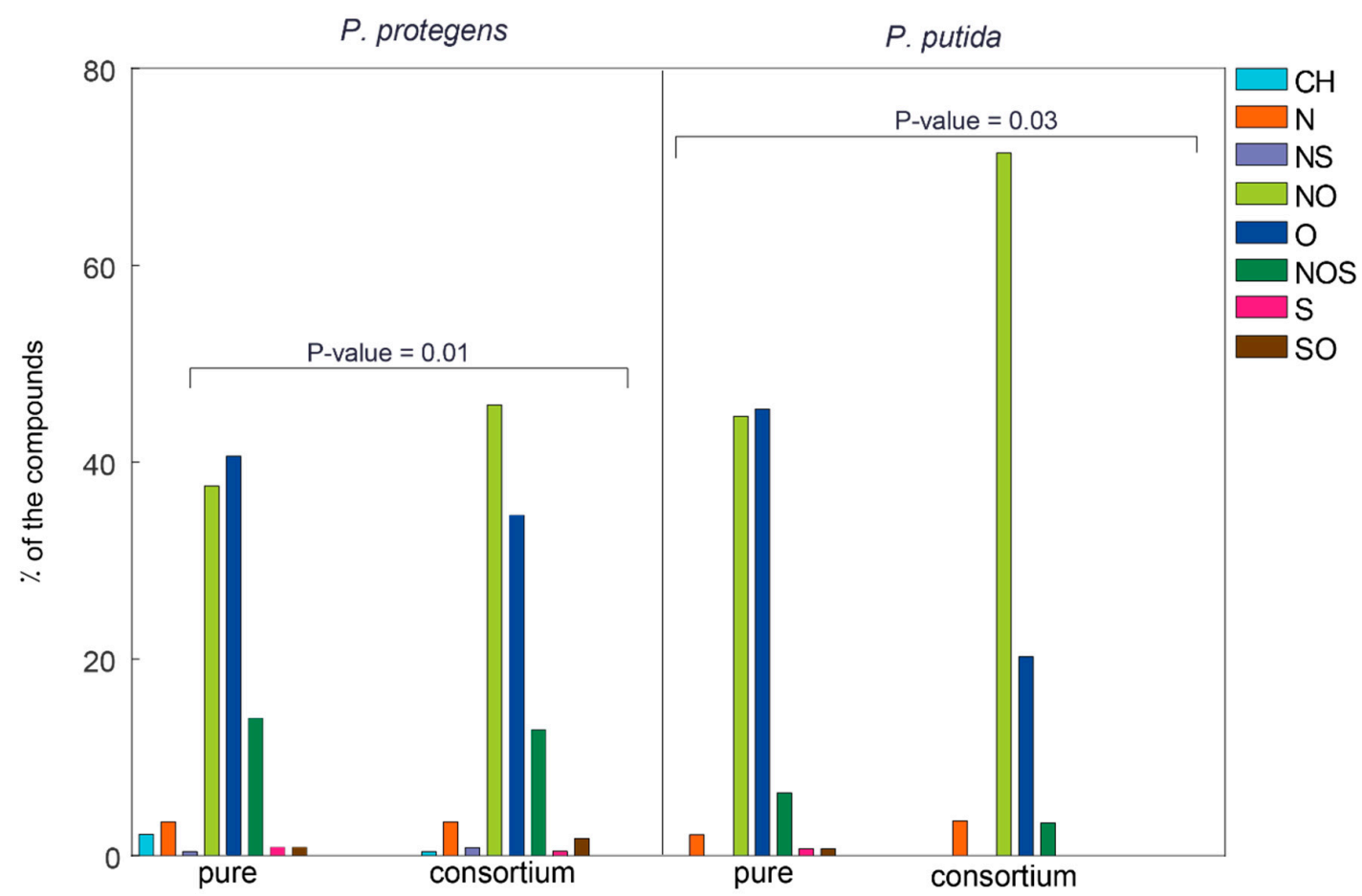

Figure 4. Heteroatomic classification of the substrates of the enzymes expressed by P. protegens and P. putida. NAFCs are classified as $\mathrm{CH}, \mathrm{N}_{\mathrm{n}}, \mathrm{N}_{\mathrm{n}} \mathrm{S}_{\mathrm{s}}, \mathrm{N}_{\mathrm{n}} \mathrm{O}_{\mathrm{o}}, \mathrm{O}_{\mathrm{o}}, \mathrm{N}_{\mathrm{n}} \mathrm{O}_{\mathrm{o}} \mathrm{S}_{\mathrm{s}}, \mathrm{S}_{\mathrm{s}}$ and $\mathrm{S}_{\mathrm{s}} \mathrm{O}_{\mathrm{o}}$ based on their heteroatomic composition. These groups have been represented as $\mathrm{CH}, \mathrm{N}, \mathrm{NS}, \mathrm{NO}, \mathrm{O}, \mathrm{NOS}, \mathrm{S}$ and $\mathrm{SO}$, respectively, in the legend. The metabolic networks of $P$. protegens and P. putida in the pure and co-cultures are significantly different. The p-values for these differences were calculated using the chi-squared test on the distribution of compounds amongst the classes. Additionally, the code used to generate this plot is published in the first Jupyter notebook that has been included in the ESI package.

We confirmed that $\mathrm{O}_{\mathrm{o}}$ and $\mathrm{N}_{\mathrm{n}} \mathrm{O}_{\mathrm{o}}$ compounds constitute two of the largest classes of NAFCs that are degraded by the microorganisms. These compounds cumulatively account for over $75 \%$ of the compounds that are degraded by the cultures. Additionally, P. protegens degrades all eight classes of the NAFCs, among which $\mathrm{CH}$ and $\mathrm{N}_{n} \mathrm{~S}_{\mathrm{s}}$ compounds are uniquely degraded by the microorganism, albeit infinitesimally. Additionally, while P. putida degrades $\mathrm{S}_{\mathrm{s}}$ and $\mathrm{S}_{\mathrm{s}} \mathrm{O}_{\mathrm{o}}$ compounds when it is cultured individually, it seems to lose this ability in the 1:1 co-culture. While we expected the metabolic networks of P. protegens and P. putida to be different, our results clearly indicate that each strain behaves differently in the pure and co-cultures.

\subsection{Characterization of the Pathways That Metabolize NAFCs}

We identified as many as 17 pathways in P. protegens that are responsible for degradation of O NAFCs when it grows in a co-culture (Figure 5). However, only two pathwaysphenylacetate degradation and arsenate detoxification-were determined to be involved in degradation the same compounds by P. putida in the co-culture. Additionally, the 3- 
oxoadipate degradation pathway is only expressed by $P$. protegens in pure cultures. This pathway was initially identified in P. putida for its conversion of catechol and protocatechuate [52]. On the other hand, the phenylacetate degradation pathway is only expressed by both strains in the co-culture, whereas the methylsalicylate pathway is expressed by pure cultures of both strains but not in the co-culture. We hypothesize that the strains utilize the methylsalicylate pathway to convert 4-methylsalicylate and 5-methylsalicylate into 4-methylcatechol, which is then degraded via a modified ortho-cleavage pathway [53], but expression of the pathway in only the pure cultures is an intriguing observation. Similarly, the cytosolic NADPH production and D-galacturonate degradation pathways are only detected in P. protegens in the 1:1 co-culture.

The metabolism of $\mathrm{N}$ and $\mathrm{NO}$ compounds could involve the participation of as many as 59 pathways in P. protegens in the 1:1 co-culture and as few as 27 pathways in pure cultures of $P$. putida. Six pathways-L-ornithine biosynthesis, nitrate reduction, purine nucleobases degradation, spermidine biosynthesis, the polyamine biosynthetic superpathway and thiosulfate disproportionation-are uniquely expressed by P. protegens in the co-culture. On the other hand, pure cultures of the same host express six unique pathways for biosynthesis of 2-aminoethylphosphonate, adenosine ribonucleotides, UDP-2,3-diacetamido-2,3dideoxy- $\alpha$-D-mannuronate, glutathione, homoglutathione, ophthalmate and 4-amino-2methyl-5-diphosphomethylpyrimidine, as well as a unique pathway for deconjugation of bile acids. The only pathway that is uniquely detected in pure cultures of P. putida is the glycine biosynthetic pathway, a single reaction pathway comprising the metabolite 5,10-methylenetetrahydropteroyl mono-L-glutamate. The NOS pathways for oxygenindependent biosynthesis of heme $\mathrm{b}$ and the biosynthesis of 3,8-divinyl-chlorophyllide were detected in all four samples. Additionally, P. protegens cells in the consortium express five unique pathways for degradation of NOS compounds, including the methylwyosine, 7-(3-amino-3-carboxypropyl)-wyosine and wybutosine biosynthetic pathways, the superpathway for polyamine biosynthesis and a pathway for 5-oxo-L-proline metabolism.

Pure cultures of $P$. protegens also uniquely express the taurine degradation pathway and the biosynthetic pathways for ergothioneine and glutathione, whereas pure cultures of P. putida uniquely express the biosynthetic pathway for deacetylcephalosporin C. Herein, isopenicillin $\mathrm{N}$ is isomerized to penicillin $\mathrm{N}$, following which the penam thiazolidine ring is oxidatively opened and expanded to yield a six-membered dihydrothiazine ring. Incidentally, the pure cultures of both strains express methylsalicylate degradation pathway. Lastly, the only unique pathways that play a role in metabolism of $\mathrm{S}$ and $\mathrm{SO}$ compounds were detected in cultures of $P$. protegens. Of these, three pathways were uniquely expressed by $P$. protegens cells in the co-culture, whereas the L-cysteine biosynthetic pathway is uniquely detected in the pure culture.

\subsection{Analysis of Differential Expression in Pure and Co-Cultures of the Pseudomonads}

We estimated the ratio between the TPM values of each pseudomonad in the 1:1 co-culture and each pure culture. The use of suppression subtractive hybridization (SSH) for generating the RNA-seq libraries ensures that the calculated TPM values for the tester samples adequately measure the up-regulated genes. Genes with a positive $\log 2$ foldchange (viz. increased TPM values in the co-culture) are enriched and probably play a greater role in metabolism of NAFC in the co-culture compared to the pure cultures, which provides useful insights on synergistic metabolism by the strains. Similarly, the MA plot for differential expression of the genes reveals that the distribution of expression is more or less symmetrical (Figure 6). We also cross-referenced the genes exhibiting the greatest changes in TPM between the 1:1 co-culture and the pure cultures of both strains in the database of KEGG pathways to shed light on how the metabolic networks of both strains adapt during co-culturing (the top five hits are listed in Tables 2 and 3, all pathways have been summarized in Table S4 in the ESI). Additionally, we have also compiled the maps of the top five pathways in Figure S2, which has been uploaded to the article's webpage as a separate file. 


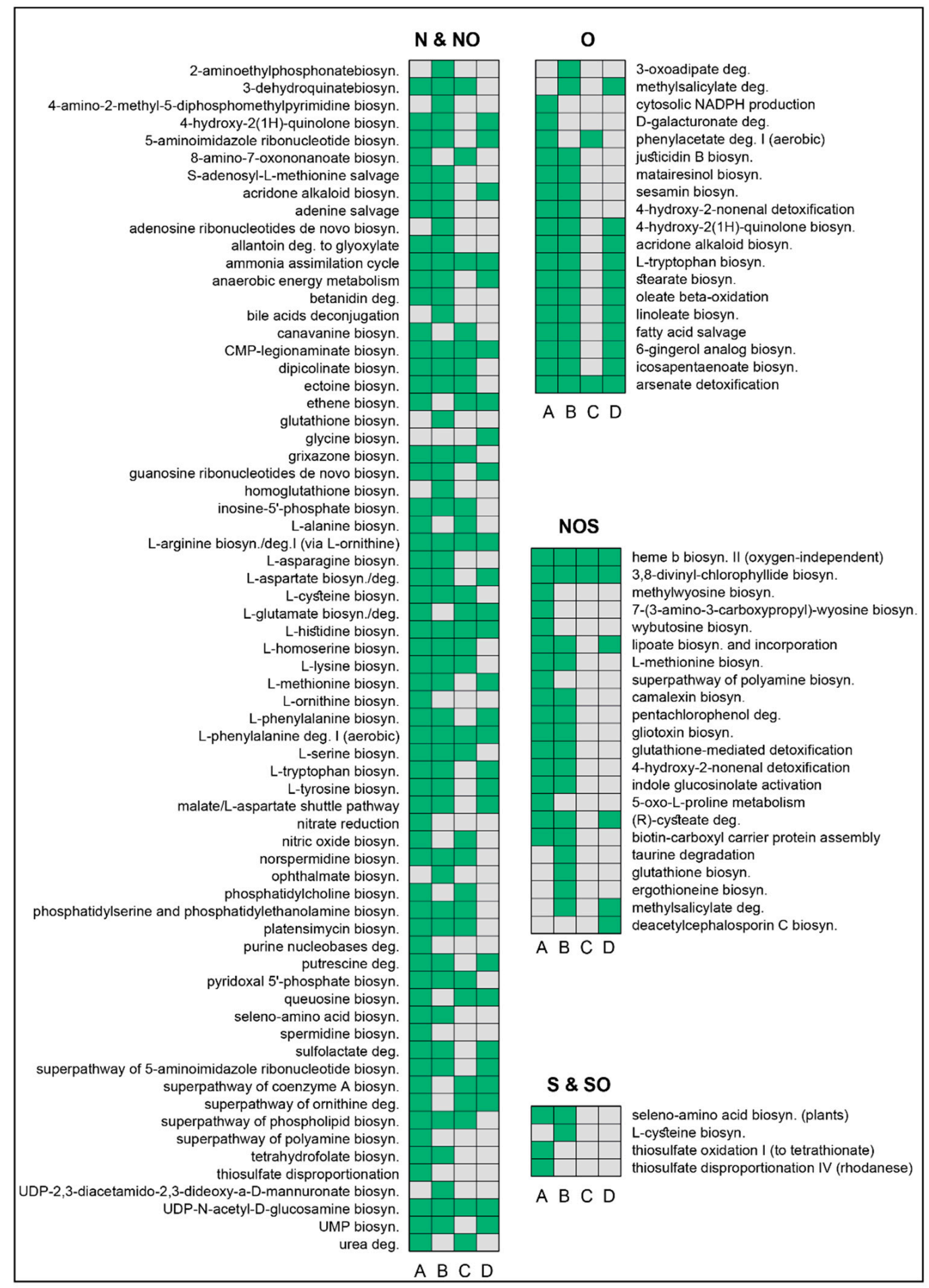

Figure 5. Metabolic pathways that are employed by the strains to degrade NAFCs. The compounds identified by matching the EC numbers of gene products in the RNA-seq libraries with enzymatic reactions in the MetaCyc database were categorized based on their heteroatomic composition into (1) N and NO, (2) O, (3) NOS and (4) S and SO classes. We subsequently determined the biodegradation pathways of these compounds by matching the chemical IDs (CID) of the substrates to EC numbers of putative enzymes and later interrogating all combinations of CID-EC combinations in the entire MetaCyc database. We identified pathways for (A) P. protegens in the 1:1 co-culture, (B) P. protegens cultured individually, (C) P. putida in the 1:1 co-culture and (D) P. putida cultured individually. Green boxes denote presence and grey boxes denote absence of a particular pathway. The codes used to generate this plot are published in the Jupyter notebooks that has been included in the ESI package. 


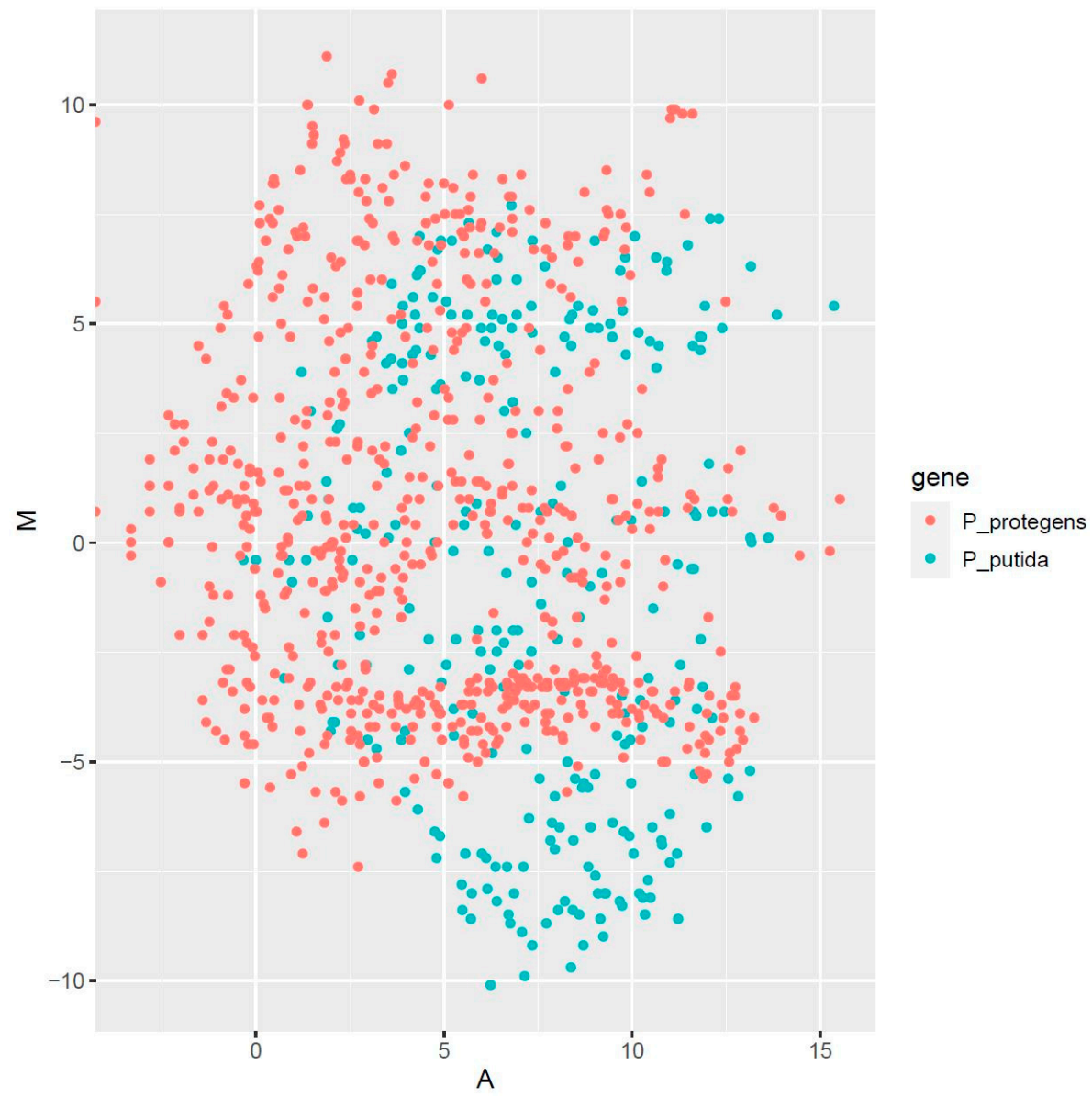

Figure 6. MA plot for differential expression of the genes. $\mathrm{M}$ is estimated as $\log _{2}$ (TPM of the consortium/TPM of the individual strains), whereas A is determined as $0.5 \times \log _{2}$ (TPM of the consortium $\times$ TPM of the individual strains). These values are calculated for all up-regulated genes.

Table 2. KEGG hits for pathways expressed by P. protegens in the 1:1 co-culture that exhibit the greatest changes in TPM compared to its metabolic activity in pure cultures.

\begin{tabular}{|c|c|c|c|}
\hline \multicolumn{4}{|c|}{ Top 5 Pathways with the Greatest Increase in TPM } \\
\hline Name & ID & Enzyme Count & Genes \\
\hline Fatty acid degradation & map00071 & 8 & fadB_2, betB_2, fadD_3, mmgC_1, dmdC_5 \\
\hline Valine, leucine and isoleucine degradation & map00280 & 7 & davT_1, fadB_2, betB_2, dmdC_5, aidB, lpdG_2 \\
\hline Glycolysis/gluconeogenesis & map00010 & 6 & betB_2, aceE, ppsA, cbbA, lpdG_2 \\
\hline Tryptophan metabolism & map00380 & 6 & fadB_2, betB_2, mmgC_1, DJCOHBMJ_04543, lpdG_2 \\
\hline Amino sugar and nucleotide sugar metabolism & map00520 & 6 & wbpA, capD, rkpK_1, gtaB, glmM, gtaB \\
\hline \multicolumn{4}{|c|}{ Top 5 Pathways with the Greatest Decrease in TPM } \\
\hline Name & ID & Enzyme Count & Genes \\
\hline Purine metabolism & map00230 & 10 & $\begin{array}{l}\text { recA, uvrD, zapE_3, lepA, uvrA_2, rep_2, lon_1, } \\
\text { DJCOHBMJ_04023, apxIB, ettA, gyrB, ffh, } \\
\text { DJCOHBMJ_04017, recD, amn, cysC_1guaA, relA_2, } \\
\text { apt, DJCOHBMJ_06051, nrdB, purL, hpt }\end{array}$ \\
\hline Propanoate metabolism & map00640 & 10 & $\begin{array}{c}\text { fadJ_2, acnD, menB_1, bauC_1, accD, bkdA2, acs, } \\
\text { bauC_1, lpdG_1, sucD }\end{array}$ \\
\hline Carbon fixation pathways in prokaryotes & map00720 & 7 & fadJ_2, accD, acs, acnD, fumC_1, sucD \\
\hline Valine, leucine and isoleucine degradation & map00280 & 6 & fadJ_2, menB_1, bauC_1, bkdA2, lpdG_1 \\
\hline Drug metabolism—other enzymes & map00983 & 6 & yfcG_2, gstB_1, guaA, rnk_1, ileS, nrdB, hpt \\
\hline
\end{tabular}


Table 3. KEGG hits for pathways expressed by P. putida in the 1:1 co-culture that exhibit the greatest changes in TPM compared to its metabolic activity in pure cultures.

\begin{tabular}{cccc}
\hline \multicolumn{1}{c}{ Top 5 Pathways with the Greatest Increase in TPM } & \\
\hline Name & ID & Enzyme Count & Genes \\
\hline Pyruvate metabolism & map00620 & 5 & maeB \\
\hline Glutathione metabolism & map00480 & 3 & ldc, ggt_1, ggt_1 \\
\hline Aminoacyl-tRNA biosynthesis & map00970 & 3 & metG, lysS, ndk \\
\hline Arginine and proline metabolism & map00330 & 3 & ldc, map_1, HNFJGDPB_03903 \\
\hline Lysine biosynthesis & map00300 & 2 & dapL \\
\hline Name & Top 5 Pathways with the Greatest Decrease in TPM & Genes \\
\hline Glycolysis/Gluconeogenesis & map00010 & 4 & pckA, pfkB \\
\hline Pyruvate metabolism & map00620 & 3 & pckA, hchA \\
\hline Arginine biosynthesis & map00220 & 3 & gdhB, glnA \\
\hline Amino sugar and nucleotide sugar metabolism & map00520 & 3 & wbpI, pfkB \\
\hline Nitrogen metabolism & map00910 & 3 & gdhB, glnA \\
\hline
\end{tabular}

\section{Discussion}

We investigated the transcriptomic responses of pure and 1:1 co-culture of two pseudomonads, P. protegens and P. putida after exposure to a concentrated mixture of NAFCs extracted from OSPW. Both strains were isolated from OSPW.

Unlike previous studies on microbial degradation of OSPW that solely focused on either quantifying the composition of the microbial consortium before and after degradation of NAFCs or confirming specific metabolic activities in the native microbiome of OSPW or deciphering the mechanisms of biodegradation of individual model NAFCs by varying their structures $[37,54,55]$, our investigation is the first of its kind to co-analyze transcriptomic, metabolite and toxicity data and elucidate the metabolic networks involved in degradation of all heteroatom classes of NAFCs by pure and co-cultures of the two strains. We established an entirely novel bioinformatics workflow to achieve this objective and deduced that the molecular distribution of the substrates that are degraded by both pure cultures and the 1:1 co-culture are significantly different.

We also confirmed that the 1:1 co-culture degrades a higher quantity of NAFCs compared to pure cultures of the two pseudomonads. The co-culture also completely detoxifies OSPW after 30 days of treatment. Additionally, P. protegens showed an over-representation of fatty acids, valine, leucine and isoleucine degradation pathways, which can be relevant to the degradation of linear NAs and nitrogen containing NAFCs, respectively. Our analysis also revealed that GO terms such as carbohydrate metabolism, oxidoreductase activity, purine biosynthesis and metabolic pathways involved in processing macromolecules, heterocyclic compounds and organic cyclic compounds are significantly over-represented in the samples. The data also indicate that the strains employ a variety of oxidoreductases, hydrolases and ligases to metabolize the NAFCs. Finally, our experimental observation that the 1:1 co-culture is stable is corroborated by the KEGG pathway analysis, which reveals how the metabolic network for NAFCs is shared between the two strains. Our findings indicate that the potential co-operative interaction between isolated P. protegens and P. putida can be further extended to biodegradation of more NAFCs.

In closing, our work represents a significant advance for the field of microbial bioremediation that opens previously unavailable opportunities to rationally engineer or adaptively evolve more strains with improved phenotypic traits. In particular-and courtesy of the mainstreaming of metagenomic screening-one could replace rate-limiting enzymes in the identified degradation pathways with heterologues that exhibit higher $\mathrm{k}_{\mathrm{cat}} / \mathrm{K}_{\mathrm{m}}$ values. 
Enzyme engineering too can be employed to alter an enzyme's substrate specificity, cofactor dependency, enhance $\mathrm{k}_{\mathrm{cat}}$ and/or lower $\mathrm{K}_{\mathrm{m}}$. In light of the poor solubility of NAs in water, we believe that achieving lower $\mathrm{K}_{\mathrm{m}}$ values is arguably more important than achieving higher $\mathrm{k}_{\text {cat }}$ values since microbial growth in tailings ponds will also be constrained by the availability of iron, phosphate and nitrogen, among other variables. To that end, one could employ adaptive laboratory evolution (ALE) to selectively improve the phenotype of environmental strains by culturing them in concentrated mixtures of NAs in a chemostat or batch reactor for prolonged durations [56]. ALE traces its roots to the pioneering work by Senior et al., who successfully employed the method to evolve P. putida to express a more active dehalogenase, thereby permitting it to grow on the herbicide Dalapon [57].

Supplementary Materials: The following are available online at https:/ / www.mdpi.com/article/10 $.3390 /$ microorganisms9102124/s1. The accompanying ESI package includes (1) characterization of the NAFCs using HPLC-Orbitrap; (2) genome sequencing and assembly; (3) heatmaps of NAFCs after degradation by pure cultures of P. putida, P. protegens and the 1:1 co-culture (Figure S1); (4) genome assembly statistics for P. protegens and P. putida (Table S1); (5) summary of sequencing of the RNA-seq libraries (Table S2); (6) all enriched GO terms for the upregulated genes in P. protegens, P. putida, and the 1:1 co-culture (Table S3); (7) KEGG pathway mapping results of the genes showing an increase and decrease in TPM counts for P. protegens and P. putida in the 1:1 co-culture compared to their pure cultures (Table S4); (8) KEGG pathway maps for the results summarized in Table S4 (Figure S2); and (9) Python codes, input and output files to generate the substrates of the enzymes related to NAFCs and characterize the biodegradation pathways as an ancillary zip file. Figure S2 has been uploaded as a separate file.

Author Contributions: Conceptualization, P.C., B.G. and V.G.Y.; Data curation, P.C., S.F., K.P. and V.G.Y.; Formal analysis, P.C., S.F., K.P., J.H. and V.G.Y.; Funding acquisition, B.G. and V.G.Y.; Investigation, P.C., K.P. and V.G.Y.; Methodology, P.C., K.P., J.H. and V.G.Y.; Project administration, V.G.Y.; Resources, D.M. and V.G.Y.; Software, P.C. and S.F.; Supervision, J.H., B.G. and V.G.Y.; Validation, P.C., S.F., J.H. and V.G.Y.; Visualization, P.C., S.F. and J.H.; Writing-original draft, P.C. and V.G.Y.; Writing-review \& editing, P.C., S.F., K.P., J.H., D.M., B.G. and V.G.Y. All authors have read and agreed to the published version of the manuscript.

Funding: This research was funded by Genome British Columbia [UPP034] and Allonnia L.L.C.

Institutional Review Board Statement: Not applicable.

Informed Consent Statement: Not applicable.

Data Availability Statement: All raw short and long reads and RNA-seq reads have been deposited to the NCBI Sequence Read Archive (SRA). The deposited data can be accessed through the NCBI BioProject PRJNA669150.

Acknowledgments: Parisa Chegounian acknowledges the Natural Sciences and Engineering Research Council of Canada (NSERC) for the Alexander Graham Bell Doctoral Scholarship. The authors are also thankful to David Young, James Wells and David Goertsen for their technical inputs and assistance in project management and experiments.

Conflicts of Interest: Bryne Gramlich is affiliated with Allonnia L.L.C., an environmental biotechnology company that is developing a bioremediation technology for treating OSPW. The findings described in this manuscript are the subject of a patent application by Vikramaditya G. Yadav and Parisa Chegounian. The other authors do not have any conflicts to declare.

\section{References}

1. Ahad, J.; Pakdel, H.; Gammon, P.; Mayer, B.; Savard, M.; Peru, K.; Headley, J. Distinguishing Natural from Anthropogenic Sources of Acid Extractable Organics in Groundwater near Oil Sands Tailings Ponds. Environ. Sci. Technol. 2020, 54, 2790-2799. [CrossRef]

2. Lightbown, V. New SAGD Technologies Show Promise in Reducing Environmental Impact of Oil Sand Production. J. Environ. Solut. Oil Gas Min. 2015, 1, 47-58. [CrossRef]

3. Scarlett, A.G.; Reinardy, H.C.; Henry, T.B.; West, C.E.; Frank, R.A.; Hewitt, L.M.; Rowland, S.J. Acute Toxicity of Aromatic and Non-Aromatic Fractions of Naphthenic Acids Extracted from Oil Sands Process-Affected Water to Larval Zebrafish. Chemosphere 2013, 93, 415-420. [CrossRef] 
4. Morandi, G.D.; Wiseman, S.B.; Guan, M.; Zhang, X.W.; Martin, J.W.; Giesy, J.P. Elucidating Mechanisms of Toxic Action of Dissolved Organic Chemicals in Oil Sands Process-Affected Water (OSPW). Chemosphere 2017, 186, 893-900. [CrossRef]

5. Morandi, G.D.; Wiseman, S.B.; Pereira, A.; Mankidy, R.; Gault, I.G.M.; Martin, J.W.; Giesy, J.P. Effects-Directed Analysis of Dissolved Organic Compounds in Oil Sands Process-Affected Water. Environ. Sci. Technol. 2015, 49, 12395-12404. [CrossRef] [PubMed]

6. Li, C.; Fu, L.; Stafford, J.; Belosevic, M.; Gamal El-Din, M. The Toxicity of Oil Sands Process-Affected Water (OSPW): A Critical Review. Sci. Total Environ. 2017, 601-602, 1785-1802. [CrossRef] [PubMed]

7. Loughery, J.R.; Marentette, J.R.; Frank, R.A.; Hewitt, L.M.; Parrott, J.L.; Martyniuk, C.J. Transcriptome Profiling in Larval Fathead Minnow Exposed to Commercial Naphthenic Acids and Extracts from Fresh and Aged Oil Sands Process-Affected Water. Environ. Sci. Technol. 2019, 53, 10435-10444. [CrossRef] [PubMed]

8. Headley, J.V.; Peru, K.M.; Fahlman, B.; Colodey, A.; McMartin, D.W. Selective Solvent Extraction and Characterization of the Acid Extractable Fraction of Athabasca Oils Sands Process Waters by Orbitrap Mass Spectrometry. Int. J. Mass Spectrom. 2013, 345-347, 104-108. [CrossRef]

9. Headley, J.V.; Peru, K.M.; Barrow, M.P. Advances in Mass Spectrometric Characterization of Naphthenic Acids Fraction Compounds in Oil Sands Environmental Samples and Crude Oil-A Review. Mass Spectrom. Rev. 2016, 35, 311-328. [CrossRef] [PubMed]

10. Bowman, D.T.; Jobst, K.J.; Ortiz, X.; Reiner, E.J.; Warren, L.A.; McCarry, B.E.; Slater, G.F. Improved Coverage of Naphthenic Acid Fraction Compounds by Comprehensive Two-Dimensional Gas Chromatography Coupled with High Resolution Mass Spectrometry. J. Chromatogr. A 2018, 1536, 88-95. [CrossRef]

11. Pereira, A.D.S.; Bhattacharjee, S.; Martin, J.W. Characterization of Oil Sands Process Affected Waters by Liquid Chromatography Orbitrap Mass Spectrometry. Environ. Sci. Technol. 2013, 47, 5504-5513. [CrossRef]

12. de Oliveira Livera, D.; Leshuk, T.; Peru, K.M.; Headley, J.V.; Gu, F. Structure-Reactivity Relationship of Naphthenic Acids in the Photocatalytic Degradation Process. Chemosphere 2018, 200, 180-190. [CrossRef] [PubMed]

13. Headley, J.V.; Peru, K.M.; Mohamed, M.H.; Frank, R.A.; Martin, J.W.; Hazewinkel, R.R.O.; Humphries, D.; Gurprasad, N.P.; Hewitt, L.M.; Muir, D.C.G.; et al. Chemical Fingerprinting of Naphthenic Acids and Oil Sands Process Waters-A Review of Analytical Methods for Environmental Samples. J. Environ. Sci. Health-Part A Toxic/Hazard. Subst. Environ. Eng. 2013, 48, 1145-1163. [CrossRef]

14. Zhang, L.; Zhang, Y.; El-Din, M.G. Degradation of Recalcitrant Naphthenic Acids from Raw and Ozonated Oil Sands ProcessAffected Waters by a Semi-Passive Biofiltration Process. Water Res. 2018, 133, 310-318. [CrossRef]

15. Quesnel, D.M.; Oldenburg, T.B.P.; Larter, S.R.; Gieg, L.M.; Chua, G. Biostimulation of Oil Sands Process-Affected Water with Phosphate Yields Removal of Sulfur-Containing Organics and Detoxification. Environ. Sci. Technol. 2015, 49, 13012-13020. [CrossRef]

16. Bauer, A.E.; Hewitt, L.M.; Parrott, J.M.; Bartlett, A.J.; Gillis, P.L.; Deeth, L.E.; Rudy, M.D.; Vanderveen, R.; Brown, L.; Campbell, S.D.; et al. The Toxicity of Organic Fractions from Aged Oil Sands Process-Affected Wate to Aquatic Species. Sci. Total Environ. 2019, 669, 702-710. [CrossRef]

17. Quagraine, E.K.; Peterson, H.G.; Headley, J.V. In Situ Bioremediation of Naphthenic Acids Contaminated Tailing Pond Waters in the Athabasca Oil Sands Region-Demonstrated Field Studies and Plausible Options: A Review. J. Environ. Sci. Health-Part A Toxic/Hazard. Subst. Environ. Eng. 2005, 40, 685-722. [CrossRef] [PubMed]

18. Allen, E.W. Process Water Treatment in Canada's Oil Sands Industry: I. Target Pollutants and Treatment Objectives. J. Environ. Eng. Sci. 2008, 7, 123-138. [CrossRef]

19. COSIA COSIA Challenge: Passive Organics Treatment Technology. Available online: https://bit.ly/3dOJgqE (accessed on 2 April 2020).

20. Yu, X.; Lee, K.; Ma, B.; Asiedu, E.; Ulrich, A.C. Indigenous Microorganisms Residing in Oil Sands Tailings Biodegrade Residual Bitumen. Chemosphere 2018, 209, 551-559. [CrossRef]

21. Siddique, T.; Stasik, S.; Mohamad Shahimin, M.F.; Wendt-Potthoff, K. Microbial Communities in Oil Sands Tailings: Their Implications in Biogeochemical Processes and Tailings Management; McGenity, T.J., Ed.; Springer: Berlin, Germany, 2018.

22. Foght, J.M.; Gieg, L.M.; Siddique, T. The Microbiology of Oil Sands Tailings: Past, Present, Future. FEMS Microbiol. Ecol. 2017, 93, fix034. [CrossRef]

23. Xue, J.; Zhang, Y.; Liu, Y.; Gamal El-Din, M. Dynamics of Naphthenic Acids and Microbial Community Structures in a Membrane Bioreactor Treating Oil Sands Process-Affected Water: Impacts of Supplemented Inorganic Nitrogen and Hydraulic Retention Time. RSC Adv. 2017, 7, 17670-17681. [CrossRef]

24. Xue, J.; Huang, C.; Zhang, Y.; Liu, Y.; Gamal El-Din, M. Bioreactors for Oil Sands Process-Affected Water (OSPW) Treatment: A Critical Review. Sci. Total Environ. 2018, 627, 916-933. [CrossRef]

25. Martin, J.W. The Challenge: Safe Release and Reintegration of Oil Sands Process-Affected Water. Environ. Toxicol. Chem. 2015, 34, 2682. [CrossRef]

26. Del Rio, L.F.; Hadwin, A.K.M.; Pinto, L.J.; MacKinnon, M.D.; Moore, M.M. Degradation of Naphthenic Acids by Sediment Micro-Organisms. J. Appl. Microbiol. 2006, 101, 1049-1061. [CrossRef] [PubMed]

27. Hadwin, A.K.M.; Del Rio, L.F.; Pinto, L.J.; Painter, M.; Routledge, R.; Moore, M.M. Microbial Communities in Wetlands of the Athabasca Oil Sands: Genetic and Metabolic Characterization. FEMS Microbiol. Ecol. 2006, 55, 68-78. [CrossRef] [PubMed] 
28. Zhang, Y.; McPhedran, K.N.; Gamal El-Din, M. Pseudomonads Biodegradation of Aromatic Compounds in Oil Sands ProcessAffected Water. Sci. Total Environ. 2015, 521-522, 59-67. [CrossRef]

29. Johnson, R.J.; West, C.E.; Swaih, A.M.; Folwell, B.D.; Smith, B.E.; Rowland, S.J.; Whitby, C. Aerobic Biotransformation of Alkyl Branched Aromatic Alkanoic Naphthenic Acids via Two Different Pathways by a New Isolate of Mycobacterium. Environ. Microbiol. 2012, 14, 872-882. [CrossRef]

30. Alberta Energy Regulator. Directive 085: Fluid Tailings Management for Oil Sands Mining Projects; Alberta Energy Regulator: Calgary, AB, Canada, 2017.

31. Quinlan, P.J.; Tam, K.C. Water Treatment Technologies for the Remediation of Naphthenic Acids in Oil Sands Process-Affected Water. Chem. Eng. J. 2015, 279, 696-714. [CrossRef]

32. Chegounian, P.; Zerriffi, H.; Yadav, V.G. Engineering Microbes for Remediation of Oil Sands Tailings. Trends Biotechnol. 2020, 38, 1192-1196. [CrossRef]

33. Wilde, M.J.; Rowland, S.J. Naphthenic Acids in Oil Sands Process Waters: Identification by Conversion of the Acids or Esters to Hydrocarbons. Org. Geochem. 2018, 115, 188-196. [CrossRef]

34. Aitken, C.M.; Head, I.M.; Jones, D.M.; Rowland, S.J.; Scarlett, A.G.; West, C.E. Comprehensive Two-Dimensional Gas Chromatography-Mass Spectrometry of Complex Mixtures of Anaerobic Bacterial Metabolites of Petroleum Hydrocarbons. J. Chromatogr. A 2018, 1536, 96-109. [CrossRef]

35. Rowland, S.J.; Scarlett, A.G.; Jones, D.; West, C.E.; Frank, R.A. Diamonds in the Rough: Identification of Individual Naphthenic Acids in Oil Sands Process Water. Environ. Sci. Technol. 2011, 45, 3154-3159. [CrossRef] [PubMed]

36. Han, X.; Scott, A.C.; Fedorak, P.M.; Bataineh, M.; Martin, J.W. Influence of Molecular Structure on the Biodegradability of Naphthenic Acids. Environ. Sci. Technol. 2008, 42, 1290-1295. [CrossRef]

37. Misiti, T.M.; Tezel, U.; Pavlostathis, S.G. Effect of Alkyl Side Chain Location and Cyclicity on the Aerobic Biotransformation of Naphthenic Acids. Environ. Sci. Technol. 2014, 48, 7909-7917. [CrossRef]

38. Blakley, E.R. The Microbial Degradation of Cyclohexanecarboxylic Acid: A Pathway Involving Aromatization to Form pHydroxybenzoic Acid. J. Microbiol. 1974, 20, 1297-1306. [CrossRef]

39. Wang, X.; Chen, M.; Xiao, J.; Hao, L.; Crowley, D.E.; Zhang, Z.; Yu, J.; Huang, N.; Huo, M.; Wu, J. Genome Sequence Analysis of the Naphthenic Acid Degrading and Metal Resistant Bacterium Cupriavidus Gilardii CR3. PLoS ONE 2015, 10 , e0132881. [CrossRef]

40. Johnson, R.J.; Smith, B.E.; Sutton, P.A.; McGenity, T.J.; Rowland, S.J.; Whitby, C. Microbial Biodegradation of Aromatic Alkanoic Naphthenic Acids Is Affected by the Degree of Alkyl Side Chain Branching. ISME J. 2011, 5, 486-496. [CrossRef]

41. Scott, A.C.; MacKinnon, M.D.; Fedorak, P.M. Naphthenic Acids in Athabasca Oil Sands Tailings Waters Are Less Biodegradable than Commercial Naphthenic Acids. Environ. Sci. Technol. 2005, 39, 8388-8394. [CrossRef] [PubMed]

42. Franzosa, E.A.; Hsu, T.; Sirota-Madi, A.; Shafquat, A.; Abu-Ali, G.; Morgan, X.C.; Huttenhower, C. Sequencing and beyond: Integrating Molecular "omics" for Microbial Community Profiling. Nat. Rev. Microbiol. 2015, 13, 360-372. [CrossRef]

43. Huang, N.; Mao, J.; Zhao, Y.; Hu, M.; Wang, X. Multiple Transcriptional Mechanisms Collectively Mediate Copper Resistance in Cupriavidus Gilardii CR3. Environ. Sci. Technol. 2019, 53, 4609-4618. [CrossRef] [PubMed]

44. Chegounian, P.; Yadav, V.G. Biodegradation of Toxic Organic Compounds in Contaminated Environments. U.S. Patent 2020/0318163 A1, 8 October 2020.

45. Seemann, T. Prokka: Rapid Prokaryotic Genome Annotation. Bioinformatics 2014, 30, 2068-2069. [CrossRef] [PubMed]

46. Thomas, K.V.; Langford, K.; Petersen, K.; Smith, A.J.; Tollefsen, K.E. Effect-Directed Identification of Naphthenic Acids as Important in Vitro Xeno-Estrogens and Anti-Androgens in North Sea Offshore Produced Water Discharges. Environ. Sci. Technol. 2009, 43, 8066-8071. [CrossRef] [PubMed]

47. Diatchenko, L.; Lau, Y.F.; Campbell, A.P.; Chenchik, A.; Moqadam, F.; Huang, B.; Lukyanov, S.; Lukyanov, K.; Gurskaya, N.; Sverdlov, E.D.; et al. Suppression Subtractive Hybridization: A Method for Generating Differentially Regulated or Tissue-Specific CDNA Probes and Libraries. Proc. Natl. Acad. Sci. USA 1996, 93, 6025-6030. [CrossRef] [PubMed]

48. Li, H.; Durbin, R. Fast and Accurate Short Read Alignment with Burrows-Wheeler Transform. Bioinformatics 2009, 25, 1754-1760. [CrossRef]

49. Quinlan, A.R.; Hall, I.M. BEDTools: A Flexible Suite of Utilities for Comparing Genomic Features. Bioinformatics 2010, 26, 841-842. [CrossRef]

50. Hunter, S.; Jones, P.; Mitchell, A.; Apweiler, R.; Attwood, T.K.; Bateman, A.; Bernard, T.; Binns, D.; Bork, P.; Burge, S.; et al. InterPro in 2011: New Developments in the Family and Domain Prediction Database. Nucleic Acids Res. 2012, 40, 306-312. [CrossRef]

51. Yu, G.; Wang, L.G.; Han, Y.; He, Q.Y. ClusterProfiler: An R Package for Comparing Biological Themes among Gene Clusters. OMICS A J. Integr. Biol. 2012, 16, 284-287. [CrossRef]

52. Ornston, L.; Stanier, R. The Conversion of Catechol and Protocatechuate to Beta-Ketoadipate by Pseudomonas Putida. J. Biol. Chem. 1966, 241, 3776-3786. [CrossRef]

53. Cámara, B.; Bielecki, P.; Kaminski, F.; Dos Santos, V.M.; Plumeier, I.; Nikodem, P.; Pieper, D.H. A Gene Cluster Involved in Degradation of Substituted Salicylates via Ortho Cleavage in Pseudomonas Sp. Strain MT1 Encodes Enzymes Specifically Adapted for Transformation of 4-Methylcatechol and 3-Methylmuconate. J. Bacteriol. 2007, 189, 1664-1674. [CrossRef] 
54. Zhang, L.; Zhang, Y.; Patterson, J.; Arslan, M.; Zhang, Y.; Gamal El-Din, M. Biofiltration of Oil Sands Process Water in Fixed-Bed Biofilm Reactors Shapes Microbial Community Structure for Enhanced Degradation of Naphthenic Acids. Sci. Total Environ. 2020, 718, 137028. [CrossRef]

55. Friedman, J.; Higgins, L.M.; Gore, J. Community Structure Follows Simple Assembly Rules in Microbial Microcosms. Nat. Ecol. Evol. 2017, 1, 0109. [CrossRef] [PubMed]

56. Guzmán, G.I.; Sandberg, T.E.; LaCroix, R.A.; Nyerges, Á.; Papp, H.; de Raad, M.; King, Z.A.; Hefner, Y.; Northen, T.R.; Notebaart, R.A.; et al. Enzyme Promiscuity Shapes Adaptation to Novel Growth Substrates. Mol. Syst. Biol. 2019, 15, e8462. [CrossRef] [PubMed]

57. Senior, E.; Bull, A.; Slater, J. Enzyme Evolution in a Microbial Community Growing on the Herbicide Dalapon. Nature 1976, 263, 476-479. [CrossRef] [PubMed] 